\title{
Analysis of Low frequency Oscillation in Power System
}

\author{
Xiaorui CHU \\ School of Engineering and Technology, Xichang College, Sichuan Xichang, 615013, China \\ e-mail: swjtucxr@163.com
}

\begin{abstract}
Around the type of power system of the low frequency oscillation, the mechanism of production, the analysis methods, the analysis tools and suppression method, etc, it summarized the research status of low frequency oscillation of power system. Therefore, based on this research status, the future analysis methods and developmental trend of the low frequency oscillation of power system were discussed.
\end{abstract}

Keywords-power system; low frequency oscillation; disturbance; mechanism

In the power system, the angle of attack among generators, the voltage on the nodal points and the tidal current of junctor and so on had the constant-amplitude or the increased range vibrates, and the oscillation frequency (generally is $0.2 \sim 2.5 \mathrm{~Hz}$ ) is lower than the system frequency. This oscillation form is called the low frequency oscillation ${ }^{[1]}$. Then the frequency of the low frequency oscillation is low, cycle to be longer,and affected area to be wide, it is also big harm to the power system. Therefore, at present, studying low frequency vibration has the even more important meaning ${ }^{[2]}$.

\section{The Types of LOW FREQUenCy OSCILLATION OF THE POWER SYSTEM}

According to the scope of the oscillation frequency and size of low frequency oscillation of power system, it can be divided into two types: Local oscillation mode (local mode) and regional oscillation mode (inter-area mode). Local oscillation mode is a generator in the area or electrical close to a few generators relative area the rest of the generator oscillation, the oscillation frequency is generally higher, between $0.7 \mathrm{~Hz}$ and $2.5 \mathrm{~Hz}$, the oscillation limits in the area, and affects the small area. Regional oscillation mode is oscillation for each other between the two sets in different areas of system, and the range of frequency is $0.2 \sim 0.7 \mathrm{~Hz}$. This oscillation has greater harmful. Once it happened, it will spread to the whole system through junctor. ${ }^{[3]}$

\section{The MEChanism of LOW Frequency OsCILlation of POWER SYSTEM}

\section{A. Negative Damping Mechanism}

Due to the increase of enlargement factor of the excitation system, the real part of numerical value of characteristic root which corresponds with the rotor mechanical

This paper is supported by Sichuan educational committee natural science foundation for youths (11ZB112). oscillations is changed by the positive, which produces the negative damping effect and counterbalances positive damping, making total damping of the system negative or very small.Once the disturbance appears, the system shafting will turn up increasing oscillation, initiating low frequency vibration of the system.

\section{B. Resonance Mechanism}

Inputting or disturbing signal of the system have some kinds of characteristic relationship with natural frequency of system, it may cause resonance when it represents the low frequency oscillationis in low frequency area.

\section{Sympathetic Vibration Mechanism}

The power system is influenced by periodic disturbance outside, and when the disturbance frequency and natural frequency of system has some kinds of special relationships, it will have sympathetic vibration. When it is in the low frequency zone, it represents the low frequency vibration.

\section{Chaotic Phenomenon}

The chaos is a very complex phenomenon that cause by various parametric interactions in the nonlinear system. It is one kind of long dynamic behavior that is very sensitive to initial conditions.It often presents continuous ruleless oscillationthe following operational factor of system in the power system.

\section{E Branch Theory}

Because of the non-linear and strange phenomenon system, the non-linear branch is possible to causes the low frequency vibration of increased range ${ }^{[4]}$.

\section{ANALYTIC METHOD OF THE LOW FREQUENCY OSCILLATION OF POWER SYSTEM}

\section{A. Complete Characteristic Value Analytic Method of Low Frequency Oscillation}

First, form linear equation of state of the entire system through the transformation. Then find the all eigenvaluesuse of system by using Q-R converter technique. Finally, distinguish the mode of low frequency oscillation according to characteristic value, so we can get the running status of system. This is the complete characteristic value analytic method of low frequency oscillation.

\section{B. Selective Modal Analysis of Low Frequency oscillation}


The basic thought of selective modal analysis SMA (selective modal analysis) is greatly reduce the order of state equation by retaining state variables about low frequency oscillation and eliminating another state variables. Then solve the mode and modality of the low frequency oscillation by using the iterative method.

C. Analytic Method of Characteristic Value of all Dimensional Part of Low Frequency Oscillation

This method is to transform the coefficient matrix of differential equations of entire system into another matrix which is the same with dimension. Then get the characteristic value which is arranged in order-increasing or decreasing progressively according to the mode through the appropriate computational method. Finally, we can get the eigenvalues we care about through the inversetransform.

\section{E. Autoexcitation Analytic Method of Low FreQuency Oscillation}

The basic thought of autoexcitation analytic method is that choose a kind of machine as a self-excited machine machine in the studied system, change the state variable as reserved variable, but the another parts of the system will carry on equal, so we can get a equivalent "second-order" system, and it is easily to get the characteristic root of this "second-order" system through the method of iterative method.

\section{F. Analytic Theory of Hopf Bifurcation of the Low Frequency Oscillation}

We need solve a problem when the Hopf bifurcation happened which is the direction when the bifurcation occurred and whether the system is stable after the difference, but they are decided by the transverse condition and buckling factor.

\section{G. Emulational Analytic Method of Time Domain of Low Frequency Oscillation}

Simulation of the time domain is a mutative situation of the time domain when the system is disturbed some conditions with the aid of the computer and base on numerical analysis.

\section{H. Analytic Method Based on Measuration of the Low Frequency Oscillation}

Analytic method based on measuration is directly to analyse the signal for the waveform of data in the recordonspot, and recognize the information of oscillation mode of the system.

\section{Analytic Method Based on the Angle of Energy}

This method is analytical investigation of low frequency oscillation from the energy. However, by now, this method haven't apply in the realistic project successfully. It waits for a further research ${ }^{[5]}$.

\section{ANALYTIC TOOLS OF LOW FREQUENCY OSCILlation}

In the early days of the PSSE, the characteristic value of system is insetted into a small program named LDA (linear dynamic analysis); and updated PSSE makes this procedure become one software named EA(eigenvalue analysis) which focus on doing analysis of small perturbation.The computational method of the procedural characteristic value includes the QR algorithm, the subspace iteration and iterative method of dominant pole and so on. Under the unified modal analytic tool, it can carry on the computations and outputs of several targets. SSAP (small signal stability analysis package) is the small signal stability analytic software package that developed by Shanghai Jiaotong University and China Electric Power Research Institute. This procedure is the first time to apply IRA(implicit restart arnoldi) algorithm into the resolving characteristic value of the power system. It makes the problem of computational scale can be solved, and it can guarantee not to leak the root in the hunting zone. Using this procedural to analyse the problem of the low frequency oscillation of southern electrical network, it also has obtained a good result. At present, this procedure largely used in the south electrical network and east China electrical network. SSAT (small signal analysis tool) is the small signal analytic tool developed by Canadian Power Tech Lab. This procedure uses the QR algorithm based on the Intel Math Kernel Library, and it can carry on the computation of the entire characteristic value for about 3000 state variables. It can analyse low damping of system.and evaluate the damping question of system. It also can design and adjust controller and evaluate the safety of system online. We can use SSAT to analyze the power oscillation of Hebei security from the energy, to explain the low frequency oscillation by resonance mechanism which negative damping cannot explain $^{[5]}$

Besides the above 3 professional commercial analytic programs of low frequency oscillation, there are some softwares based on MATLAB. PSAT (power system analysis toolbox) is a analytic software of the power system developed by Dr. FedericoMilano from Hot Yarra University in Italy. and based on MATLAB, this software function is rich, graphical interface, and can be used as a small signal low frequency oscillation Analysis ${ }^{[6]}$.

\section{Method of RESTRAINING LOW FREQUENCY OSCILLATION OF POWER SYSTEM}

\section{A. Static Var Compensator(SVC)}

SVC is the wattless power source that can adjust fast, and it can provide the damping moment through outputting the variable conductance by itself. The major function is to guarantee the dynamic reactive power can adjust fast, and it also have the support function of voltage when some accidents happened suddenly, and sustain the level of 
voltage, and make thevibration of the system subside and so on.

\section{B. Thyristor Controlled Series Compensator (TCSC)}

(Thyristor controlled series compensator)TCSC of conventional mechanical control have generally applied in the tidal current control. This kind of controller was attached importance to people immediately when the concept of FACTS was proposed. Because it directly enters transmission line, it can widely adjust the line positive sequence reactance, and enhance the stability of the system, and overcome the power oscillation and subsynchronous oscillation to raise the feeding power.

\section{Thyristor Controlled Phase Shitter(TCPS)}

(Thyristor controlled phase shitter)TCPS enhances the maximal power which the line delivers and reduces the damped oscillation through controlling the angle of wiring. Traditional mechanical phase shifter only can classification adjustment, and there are not many classifications. The kinetic speed is very slow, and its life is short, and it has no ability to restrain the oscillation and improve the dynamic property of the system. However, the speed of the controllable phase shifter is fast, and its life is not influence by the number of operations.

\section{Unified Power Flow Controller(UPFC)}

The (unified power flow controller) UPFC is the third generation equipment of FACTS of series connection which is adviced by American EPRI . It makes a alternating voltage produced by a transverter thyristor joins and superimposes the phase voltage of the transmission line, and its amplitude and phase anglecausing can continuously change, so as to realize the accurate adjustment of active power and reactive power of lines, and can improve the capacity of the transmission and damping vibration of the system.

\section{E. Battery Energy Storage System and SuperConducting Magnetic Storage (BESS and SMES)}

The new equipments of restraining low frequency oscillation, such as the battery stored energy system (battery energy storage system) and the superconducting magnetism stored energy (superconducting magnetic storage) and so on are still at the research stage now and do not greatly put into the industrial application. The core part of BESS is the accumulator cell and AC/DC converter that consisted by a kind of GTO. The system of energy storage of battery can be used as rotary reserve, and also can be used as the power of pitch peak and FM, or as the large-scale UPS by directly packing in the important user. At the same time, BESS also has the function of avr adjustment.

\section{F. Power System Stabilizer(PSS)}

(Power system stabilizer) PSS was earliest proposed by American scholar FP Demello and C Conco2dri. The goal of using the stabilizer of the power system is that expand the stability limit of transmission of electrical energy through strengthening the damping of system vibration by generator excitation control. It can provide the additional excitation control of positive damping. The common parameters are angular velocity, power and frequency, and mainly consist by the magnification, reduction, and lead-lag links and other corrective connections. It takes the output and terminal voltage as the input of excitation system. PSS is designed approximate linearized pattern based on layout in a balance point of the system. It has a strong pertinence and easily realize and restrain the low frequency oscillation effectively. It has widespread application ${ }^{[7]}$.

\section{G. The Modulation of HVDC Transmission System}

When AC/DC transmission line joint operation, because direct current transmission's power can be controlled fast, therefore exchange transmission line control circuit low frequency power oscillation signal is introduced to DC transmission line control circuit, can suppress the low frequency oscillation effectively. Literature 8 using directcurrent power modulation to suppressing low frequency oscillation has done the exhaustive elaboration. The application of direct-current modulating technology is especially obvious to enhancing "regional oscillation mode" damping the effect.

\section{H. Linear Optimal Excitation Control(LOEC)}

The idea is for a given linear system or in a balance of the approximate linear in the system, the paper puts forward the sum of square of state variables and control quantity to express the quadratic index, through solving Li Kati matrix equation to obtain state feedback control that causes the performance index to reach the extreme value, This method is linearity - two times - Li Kati question in essence, it is the complete combination condition quantity most superior linear, its control effect is not influenced by oscilation frequency, therefore provides good damping effect to the ultra low frequency oscillation and time synchronization oscillation.

\section{Nonlinear Optimal Excitation Control}

The basic principle is to use the nonlinear feedback and appropriate coordinate transform, in some certain conditions, exacting the nonlinear system into the linearization, can be fully charged linear system, and using linear system theory for feedback solution. Using nonlinear control law, can realize the decentralized control for between generator completely, and has robustness to the change of network parameter in the strict sense.

\section{NEW LSSUES ANALYSIS OF LOW FREQUENCY OSCILLATION}

\section{A. Analysis Method of Power System's Low Frequency Oscillation}


Due to its low frequency oscillation is complexity and randomness, and has added very major difficulty to the accurate research, the mechanism of low frequency oscillation is complex and multiplicity, that causes the analysis method and regulatory measures research results loses the unity. In the future, we can do some research in the following 3 methods.

\section{a. The Method of Modality Analytic}

The method of modality analytic that applied to power system stability has certain feasibility in this respect. It is noteworthy that the method of modality analytic is to small perturbation stability analyzes, its mathematical foundation is that describing power system characteristic nonlinear simultaneous equation is in a movement neighbor linearization, then using the linearized system theory for the corresponding computation, therefore one of the major problems that urgently awaits to be solved at present is how to use this method to analyze large disturbance caused by the low frequency oscillation.

\section{b. The Method of Synchronized Phase Angle Survey}

The method of synchronized phase angle survey not only can provide the quantity of voltage and electric current gauging in the microsecond level precision scope, but also can survey the partial frequency and the rate of change in frequency, therefore it can supply system's most direct status messages under specific operational method, may foresee: The method of synchronized phase angle survey will obtain many applications in the next few years.

\section{c. The Method of Energy Storage}

The method of energy storage is new method studied for maintaining the power system stable in recent years, it the stored energy when the energy is surplus, but emit energy when the energy and has achieved the goal of improvement systems operation efficiency and enhancing the system stability, When there is the enormous amount energy pouring into the system, how to use the control center data to realize the fast and active control of energy (the memory and release of energy); How to stored energy system modeling, thus establishes the new algorithm that contain stored energy installment; At present the question that urgently awaits to be solved.

\section{B. The Power System of the Research Direction of the Development of Low Frequency Oscillation}

The research of related power system low frequency oscillation mechanism also needs for further deepening; In view of QR algorithm that products “dimension disaster" the question, needs to seek for the new computation the algorithm for entire characteristic value or part characteristic value; As a result of the need of power system's running attention, the parsing technique of development online visualization, it can provide the convenience for the systems operation; The vigorous development of new energy and distributional electricity generation, causes stored energy system-based the system damped control strategy to be in the key growth time; Basing on research technique of probability statistics and Intelligent control and optimization techniques analysis method has also provided the new mentality for the power system small perturbation analysis ${ }^{[8]}$.

\section{CONCLUSION}

Profoundly studying of low frequency oscillation production mechanism, analytical method and braking measure and other aspects is of great significance to the steady operation of system safety. On the one hand the electrical network plan and optimized movement provide the theoretical support; On the other hand it can renew and develop the new analysis theory and analysis tool. How existing analysis theory maintains the systemic stability well is the key point; At the same time, developing new direction actively will base on the probability statistics and intelligent control optimization method applying in the low frequency oscillation that is the difficulty; Using the stored energy system into suppressing the system's low frequency oscillation has the broad prospect..

\section{REFERENCES}

[1] GUO Quan-li, "NLow frequency oscillation in power system”, Power System Protection and Control, vol.32, no.22, pp.114-119, 2008.

[2] LIU Peng-cao, "Prospect and state of study on power system low frequency oscillation and the prospect”, Jiangxi Electric Power, vol.34, no.4, pp.13-14, 2009.

[3] Tang Zhi-da, "The influence factors in low frequency osillation" , Hujian Electric Power and Electrician, vol.26, no.4, pp.10-21, 2006.

[4] Liu Chun-mei, "Study on influencing factors of low frequency oscillation of power system " , Shanxi Electric Power, vol.20, no.11, pp.12-15, 2011.

[5] ZENG Xinyi,“'Analysis of power system low-frequency oscillation”, Low Voltage Apparatus, vol.20, no.11, pp.38-43, 2011.

[6] Liu Chun-mei,"Study of low frequency oscillation of power systems based on PSS configuration ”, Shanxi Electric Power, vol.19, no.12, pp.11-15, 2010.

[7] YI Mei-xian, "Inhibition of low frequency oscillation of power system" , Science and Technology Innovation Herald, vol.28, no.6, pp.80-81, 2009.

[8] Wang Hui-zhong,“The development and prospect on the strategy of damping low frequency oscillation in power system ”, Explosion-proof Electric Machine, vol.30, no.28, pp.80-88, 2008. 